\title{
Transcriptional response of the extremophile red alga Cyanidioschyzon merolae to changes in $\mathrm{CO}_{2}$ concentrations ${ }^{2 /}$
}

\author{
Nadine Rademacher $^{\mathrm{a}, 1}$, Thomas J. Wrobel ${ }^{\mathrm{a}, 1}$, Alessandro W. Rossoni ${ }^{\mathrm{a}}$, Samantha Kurz ${ }^{\mathrm{a}}$, \\ Andrea Bräutigam $^{\mathrm{b}}$, Andreas P.M. Weber ${ }^{\mathrm{a}}$, Marion Eisenhut ${ }^{\mathrm{a}, *}$

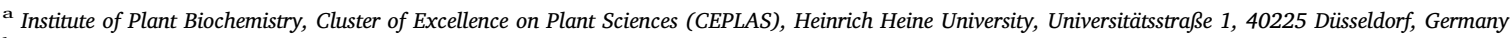 \\ b Leibniz-Institut für Pflanzengenetik und Kulturpflanzenforschung (IPK), Corrensstraße 3, 06466 Stadt Seeland, OT Gatersleben, Germany
}

\section{A R T I C L E I N F O}

\section{Keywords:}

Cyanidioschyzon merolae

RNA-seq

$\mathrm{CO}_{2}$ limitation

Photorespiration

\begin{abstract}
A B S T R A C T
Cyanidioschyzon merolae (C. merolae) is an acidophilic red alga growing in a naturally low carbon dioxide $\left(\mathrm{CO}_{2}\right)$ environment. Although it uses a ribulose 1,5-bisphosphate carboxylase/oxygenase with high affinity for $\mathrm{CO}_{2}$, the survival of $C$. merolae relies on functional photorespiratory metabolism. In this study, we quantified the transcriptomic response of $C$. merolae to changes in $\mathrm{CO}_{2}$ conditions. We found distinct changes upon shifts between $\mathrm{CO}_{2}$ conditions, such as a concerted up-regulation of photorespiratory genes and responses to carbon starvation. We used the transcriptome data set to explore a hypothetical $\mathrm{CO}_{2}$ concentrating mechanism in C. merolae, based on the assumption that photorespiratory genes and possible candidate genes involved in a $\mathrm{CO}_{2}$ concentrating mechanism are co-expressed. A putative bicarbonate transport protein and two $\alpha$-carbonic anhydrases were identified, which showed enhanced transcript levels under reduced $\mathrm{CO}_{2}$ conditions. Genes encoding enzymes of a PEPCK-type $\mathrm{C}_{4}$ pathway were co-regulated with the photorespiratory gene cluster. We propose a model of a hypothetical low $\mathrm{CO}_{2}$ compensation mechanism in $C$. merolae integrating these low $\mathrm{CO}_{2}$-inducible components.
\end{abstract}

\section{Introduction}

Photosynthetic biomass production is initialized by the fixation of one molecule of $\mathrm{CO}_{2}$ to the acceptor molecule ribulose 1,5-bisphosphate, catalyzed by the enzyme ribulose 1,5-bisphosphate carboxylase/oxygenase (Rubisco). The resulting two molecules of 3-phosphoglycerate (3-PGA) are fed into the Calvin-Benson-Bassham cycle (CCB) for reduction to carbohydrates and regeneration of the acceptor molecule. Rubisco catalyzes also an oxygenation reaction in which $\mathrm{O}_{2}$ is added to the acceptor molecule, resulting in a proportion of Rubisco occupied with the non-productive side reaction yielding one molecule of 3-PGA and one molecule of 2-phosphoglycolate (2-PG). The latter inhibits multiple essential enzymes and must hence be efficiently detoxified. The photorespiratory pathway converts two molecules of 2-PG into one molecule of 3-PGA under consumption of energy and release of $\mathrm{CO}_{2}$ and ammonia (reviewed in Bauwe et al., 2010; Hagemann et al., 2016). Two factors are critical for the rate of carboxylation versus oxygenation by Rubisco: The specificity of the enzyme for $\mathrm{CO}_{2}$ and the ratio of $\left[\mathrm{CO}_{2}\right]$ to $\left[\mathrm{O}_{2}\right]$ at the site of Rubisco. Rubisco enzymes from organisms of various photosynthetic lineages have different evolutionary origins, and differ in substrate specificity and reaction velocity. Cyanobacteria and land plants use Rubisco Form 1A and 1B, which are of cyanobacterial origin, while non-green algae, such as red algae, contain Form 1C and 1D, which are of proteobacterial origin (Hauser et al., 2015). As a consequence of the reaction mechanism, higher specificity for $\mathrm{CO}_{2}$ decreases Rubisco's velocity, while an increase in velocity is accompanied by a decline in specificity (Tcherkez et al., 2006). Cyanobacteria employ a fast Rubisco with low specificity for $\mathrm{CO}_{2}$, while land plant Rubisco achieves a higher $\mathrm{CO}_{2}$ specificity at the expense of velocity (Savir et al., 2010). Rubisco enzymes of thermophilic cyanidiophycean red algae, such as Galdieria parta and Cyanidium caldarium, exhibit the highest $\mathrm{CO}_{2}$ specificity and thus lowest velocity, measured to date (Uemura et al., 1997).

In addition to the capacities of the enzyme, the $\left[\mathrm{CO}_{2}\right]$ to $\left[\mathrm{O}_{2}\right]$ ratio next to Rubisco strongly influences the carboxylation versus oxygenation rate. To overcome this constraint, many photosynthetic organisms evolved a $\mathrm{CO}_{2}$ concentrating mechanism (CCM), which raises the $\mathrm{CO}_{2}$ concentration in close vicinity to Rubisco and thereby enhances the carboxylation rate (Giordano et al., 2005; Raven et al., 2012). The occurrence of CCMs was demonstrated for cyanobacteria, most algae

\footnotetext{
Abbreviations: CA, carbonic anhydrase; $\mathrm{CCM}, \mathrm{CO}_{2}$ concentrating mechanism; $\mathrm{HC}$, high $\mathrm{CO}_{2}$; $\mathrm{LC}$, low $\mathrm{CO}_{2}$; RNA-seq, RNA-sequencing

This article is part of a special issue entitled: Light driven reactions in model algae published at the Journal of Plant Physiology 217C.

* Corresponding author: Postal address: Institute of Plant Biochemistry, Heinrich Heine University, Universitätsstraße 1, 40225 Düsseldorf, Germany.

E-mail address: m.eisenhut@hhu.de (M. Eisenhut).

${ }^{1}$ These authors contributed equally to this work.
} 
and aquatic plants, as well as for $\mathrm{C}_{4}$ and Crassulacean acid metabolism plants (reviewed in Raven et al., 2008). The cyanobacterial CCM employs inorganic carbon $\left(\mathrm{C}_{\mathrm{i}}\right)$ uptake mechanisms for cytoplasmic bicarbonate $\left(\mathrm{HCO}_{3}{ }^{-}\right)$accumulation and specific microcompartments, the carboxysomes, which encapsulate Rubisco and carbonic anhydrase (CA). The cytoplasmic $\mathrm{HCO}_{3}{ }^{-}$diffuses into the carboxysome and is converted into $\mathrm{CO}_{2}$ by CA. Thus, the $\mathrm{CO}_{2}$ concentration is increased by a factor of up to 1000 next to Rubisco (Badger and Price, 2003; Kaplan and Reinhold, 1999). However, even the action of a CCM cannot fully repress the oxygenase activity of Rubisco (Eisenhut et al., 2006, 2008; Nakamura et al., 2005; Zelitch et al., 2009). While among aquatic photosynthetic organisms the cyanobacterial and green algal CCM are well studied, it remains unclear whether thermophilic red algae also employ such a mechanism to improve photosynthetic efficiency (Giordano et al., 2005; Zenvirth et al., 1985).

Cyanidioschyzon merolae (C. merolae) is a model organism for cyanidiophycean red algae. The $16 \mathrm{Mbp}$ genome of the single-cell organism is fully sequenced (Matsuzaki et al., 2004) and techniques for targeted gene knockout by homologous recombination as well as transient transformation for, e.g., localization studies are available (Imamura et al., 2010; Minoda et al., 2004; Watanabe et al., 2011). C. merolae tolerates temperatures up to $57{ }^{\circ} \mathrm{C}$ and prefers acidic $(\mathrm{pH}<2)$ growth medium (Seckbach, 1995). Under these conditions, $\mathrm{CO}_{2}$ is the prevalent inorganic carbon $\left(\mathrm{C}_{\mathrm{i}}\right)$ species in the aquatic environment. Although red algae use a Rubisco with high specificity for $\mathrm{CO}_{2}$ over $\mathrm{O}_{2}$, the reduced solubility of $\mathrm{CO}_{2}$ at high temperatures forces $C$. merolae to perform a plant-like photorespiratory cycle (Rademacher et al., 2016).

In this work, we quantified the transcriptional response of $C$. merolae in response to changes in $\mathrm{CO}_{2}$ concentrations by RNA-sequencing (RNA-seq) and applied the data set to predict a hypothetical CCM in $C$. merolae and to search for possible components.

\section{Material and methods}

\subsection{C. merolae cultivation}

C. merolae 10D wildtype (WT) cells were cultivated in $2 \mathrm{x}$ modified Allen's growth medium, pH 2 (Minoda et al., 2004), at $30^{\circ} \mathrm{C}$, bubbled with high $\mathrm{CO}_{2}$ concentrations $\left(5 \% \mathrm{CO}_{2}\right.$ in air, $\left.\mathrm{HC}\right)$ or low $\mathrm{CO}_{2}$ concentrations $\left(0.04 \% \mathrm{CO}_{2}\right.$ in air, LC) at $90 \mu \mathrm{mol}$ photons $\mathrm{m}^{-2} \mathrm{~s}^{-1}$ light in a Multi-Cultivator MC 1000-OD system (Photon Systems Instruments, Drasov, Czech Republic).

For the $\mathrm{CO}_{2}$ shift experiment, three independent biological replicates of continuously HC grown C. merolae WT cells were cultivated for $24 \mathrm{~h}$ under $\mathrm{HC}$ conditions with an initial optical density at $750 \mathrm{~nm}\left(\mathrm{OD}_{750}\right)$ of 0.7 . After $24 \mathrm{~h}$, cells were shifted by changing the $\mathrm{CO}_{2}$ concentration in the aeration for $24 \mathrm{~h}$ to $\mathrm{LC}$ conditions and afterwards shifted back to $\mathrm{HC}$ conditions for a $24 \mathrm{~h}$ recovery phase. For RNA extraction, $5 \mathrm{~mL}$ samples $\left(\mathrm{OD}_{750}=1.0\right)$ were taken immediately before the shift to LC (HC $0 \mathrm{~h}$ ), $3 \mathrm{~h}$ after shift to LC (LC $3 \mathrm{~h}$ ), $24 \mathrm{~h}$ after shift to LC (LC $24 \mathrm{~h}$ ), and $24 \mathrm{~h}$ after a recovery phase at HC (HC 24 h). A sampling scheme is illustrated in Fig. 1A.

\subsection{Gene expression analysis by RNA-seq}

For RNA isolation, the $5 \mathrm{~mL}$ samples were centrifuged for $5 \mathrm{~min}$ at $4{ }^{\circ} \mathrm{C}(3000 \mathrm{rpm})$. RNA extraction from the cell pellet was performed using the EURx GeneMatrix Universal RNA Purification Kit (Roboklon, Berlin, Germany) following the manufacturer's protocol for RNA cell extraction. DNA was removed by treatment with RNase-free DNaseI (New England Biolabs, Ipswich, USA).

Libraries were prepared using the TruSeq RNA Sample Prep Kit v2 (Illumina, San Diego, USA). RNA integrity, sequencing library quality and fragment size were checked on a 2100 Bioanalyzer (Agilent). Average library size was $320 \mathrm{bp}$ with equimolar pooling $(2 \mathrm{nM})$. Demultiplexed Illumina reads were aligned with RSEM (parameters: - very-sensitive; -calc-pme; -calc-ci; - gibbs-burnin 500) with the
A

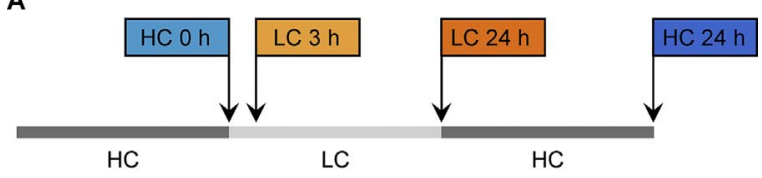

B

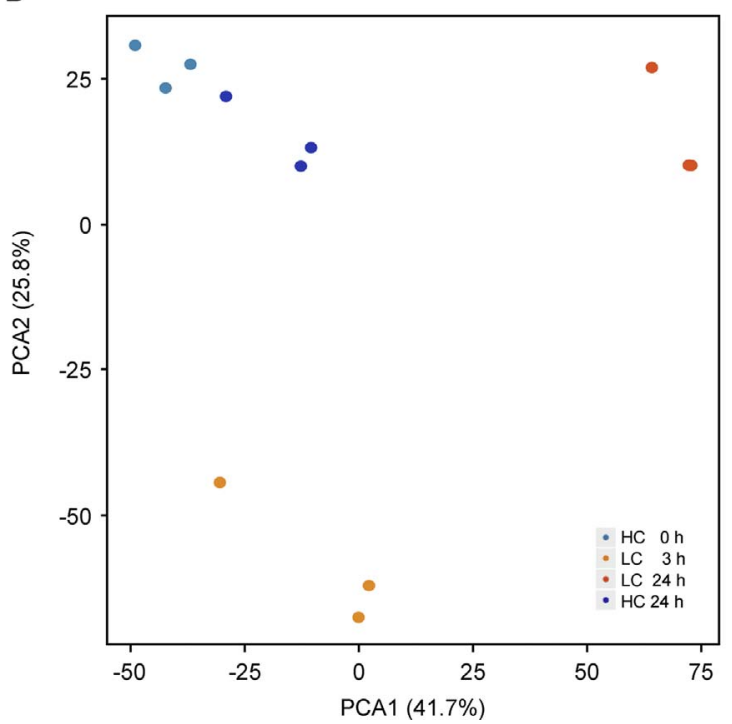

Fig. 1. Overview of the $\mathrm{CO}_{2}$ shift experiment. A. Experimental set-up of the $\mathrm{CO}_{2}$ shift experiment. C. merolae cells were cultivated under high $\mathrm{CO}_{2}\left(5 \% \mathrm{CO}_{2}\right.$ in air, $\left.\mathrm{HC}\right)$ conditions, shifted for $24 \mathrm{~h}$ to low $\mathrm{CO}_{2}\left(0.04 \% \mathrm{CO}_{2}\right.$ in air, $\left.\mathrm{LC}\right)$ conditions, and then shifted back to HC conditions. Samples for RNA-seq were taken immediately before the shift to LC (HC $0 \mathrm{~h}$ ), $3 \mathrm{~h}$ (LC $3 \mathrm{~h}$ ) and $24 \mathrm{~h}$ (LC $24 \mathrm{~h}$ ) after the shift to LC, and $24 \mathrm{~h}$ after the recovery at $\mathrm{HC}$ (HC $24 \mathrm{~h}$ ). The color code for the different samples applies for the complete study. B. Principle component analysis of RNA-seq data.

default aligner bowtie2 (Li and Dewey, 2011) to the reference transcriptome of C. merolae 10D (ASM9120v1.30.gtf) (Nozaki et al., 2007), which was retrieved from the ENSEMBL database (Yates et al., 2016).

Differential gene expression was analyzed using the EdgeR package (McCarthy et al., 2012) in R. All sequenced conditions were analyzed in a pairwise manner with $\mathrm{HC} 0 \mathrm{~h}$ as reference point. A q-value of 0.01 was chosen as significance threshold for single gene differential expression after correction for multiple testing via the Bonferroni algorithm to limit the false positive rate to close to zero at the cost of a higher false negative rate (Krzywinski and Altman, 2014). For $K$-means clustering transcripts per million (TPM) values were scaled to their average. Sum of square errors were used to determine the suitable number of clusters at 10, $\mathrm{K}$-means clustering with Euclidean distance carried out 10,000 times, and the clustering with the best SSE ratio used for further analysis. Principle component analysis was performed on scaled TPM values.

The MapMan-based functional categorization of all genes in the $C$. merolae genome was performed by comparing their protein sequence to Arabidopsis TAIR10 (http://www.arabidopsis.org/) using the standalone version of NCBI BLASTP $(2.2 .31+)$ with default settings. The MapMan categorization was transferred from TAIR10. Functional enrichment was performed on hierarchical, independent MapMan categories reduced to their first and second level using Fisheŕs exact tests. All $P$-values were corrected for multiple testing via the Benjamini Hochberg algorithm (Table S1) (Benjamini and Hochberg, 1995). GO terms for C. merolae proteins were retrieved from the Uniprot database and matched to gene identifiers using the ENSEMBL database (Yates et al., 2015). GO term enrichment was tested with the TopGO package (parameters; nodeSize $=10$, ontology $=$ "BP") (https://bioconductor.org/packages/release/bioc/html/topGo.html; Alexa and Rahnenfuhrer, 2016) in R. Statistically significant terms were calculated with classic Fishers exact test and without weighing and corrected according to Benjamini-Yekutieli to account for dependency of GO terms 

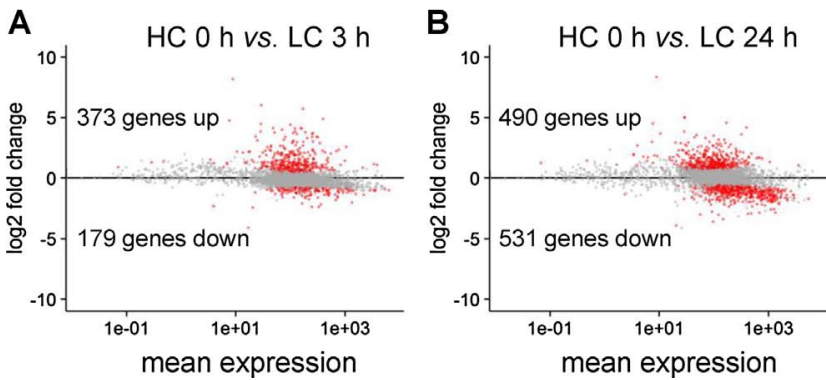

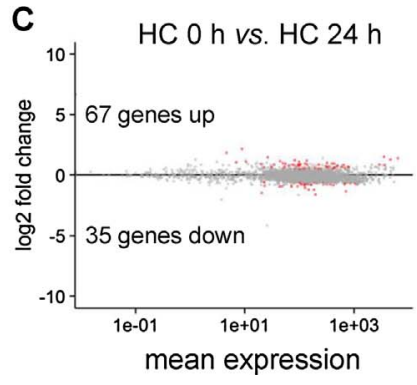

Fig. 2. Global transcriptional response of C. merolae toward changes in $\mathrm{CO}_{2}$ concentrations. A. Short-term ( $3 \mathrm{~h}$ after shift from HC to LC conditions) effects of reduced $\mathrm{CO}_{2}$ availability on gene expression. B. Longterm ( $24 \mathrm{~h}$ after shift from HC to LC conditions) effects of reduced $\mathrm{CO}_{2}$ availability on gene expression. C. Recovery effects $24 \mathrm{~h}$ after re-shift from LC to HC conditions. Changes are given as log2-fold changes compared to HC $0 \mathrm{~h}$. Significance was tested with EdgeR (q < 0.01; Robinson et al., 2010). Significantly changed genes are highlighted as red dots. Numbers of significantly up- and down-regulated genes are indicated.
(Benjamini and Yekutieli, 2001) (Table S2). Only significant enrichments ( $\mathrm{q}<0.05)$ are reported in the text. Transcription factors were annotated based on Pérez-Rodríguez et al. (2010). Heatmaps were created using the heatmap.2 package (https://cran.r-project.org/web/packages/gplots/ gplots.pdf).

The complete RNA-seq data set is provided in Table S3. The read data have been submitted to the National Center for Biotechnology Information Gene Expression Omnibus under accession number GSE100372 (http:// www.ncbi.nlm.nih.gov/geo/query/acc.cgi?acc $=$ GSE100372).

\subsection{In silico analyses of carbonic anhydrases}

Amino acid sequences of CMT416C and CMI270C were retrieved from the Cyanidioschyzon merolae Genome Project data base (http:// merolae.biol.s.u-tokyo.ac.jp) and the alignment performed with the ClustalW on the Phylogeny.fr platform (http://www.phylogeny.fr, Dereeper et al., 2008). The SOSUI platform (http://harrier.nagahama-ibio.ac.jp/sosui/, Hirokawa et al., 1998) was employed to search for transmembrane regions. TargetP1.1 (http://www.cbs.dtu.dk/services/ TargetP/, Emanuelsson et al., 2000) was used for prediction of hypothetical targeting peptides.

\subsection{Analysis of subcellular localization of carbonic anhydrases}

For subcellular localization of the carbonic anhydrases (CA) CMT416C and CMI270C in Nicotiana benthamiana ( $N$. benthamiana) protoplasts and $C$. merolae cells, respectively, two constructs were generated for each protein, fusing CMT416C and CMI270C with the yellow fluorescent protein (YFP) either at the $\mathrm{N}$ - or C-terminus. The coding sequences were amplified by PCR using C. merolae genomic DNA as template and cloned into the pUBN-YFP or pUBC-YFP vector applying gateway technology (Grefen et al., 2010). In pUBN-YFP and pUBC-YFP expression of the fusion proteins is under the control of the UBIQUTIN 10 promoter. Primer sequences are listed in Table S4. Transient transformation of $N$. benthamiana leaves was carried out using the Agrobacterium tumefaciens strain GV3101. Protoplast isolation and microscope analysis was performed $2 \mathrm{~d}$ after infiltration using a Zeiss LSM 510 Meta confocal - scanning laser microscope as described in Breuers et al., 2012. Transient transformation of $C$. merolae cells was performed as described by Ohnuma et al., 2008. Microscope analysis was perfomed $24 \mathrm{~h}$ after transformation with a Zeiss LSM 780 microscope.

\section{Results}

\subsection{Effects of reduced $\mathrm{CO}_{2}$ concentrations on transcriptome}

To analyze transcriptional changes in response to altered $\mathrm{CO}_{2}$ concentrations, we performed a $\mathrm{CO}_{2}$ shift experiment. $C$. merolae cells were cultivated for $24 \mathrm{~h}$ under $\mathrm{HC}\left(5 \% \mathrm{CO}_{2}\right.$ in air) conditions, then shifted for $24 \mathrm{~h}$ to $\mathrm{LC}$ conditions $\left(0.04 \% \mathrm{CO}_{2}\right.$ in air), and finally shifted back to HC conditions for another $24 \mathrm{~h}$. Samples were taken in biological triplicates immediately before the shift to LC (HC $0 \mathrm{~h}$ ), $3 \mathrm{~h}$ after the shift (LC $3 \mathrm{~h}$ ), $24 \mathrm{~h}$ after the shift (LC $24 \mathrm{~h}$ ), and $24 \mathrm{~h}$ after the recovery under HC conditions (HC $24 \mathrm{~h}$ ). The experimental set-up is illustrated in Fig. 1A. RNA-seq analysis generated 726,326,434 Illumina paired-end reads with about 18.4 Million paired-end reads per sample on average. $92 \%$ of the RNA-reads mapped to the reference genome of $C$. merolae (Matsuzaki et al., 2004).

To evaluate reproducibility among biological replicates, a principle component analysis (PCA) was performed on the transcripts per million (TPM) values. The biological triplicates clearly clustered together (Fig. 1B), indicating lower variation between biological replicates compared to the treatment. Furthermore, the separation of the samples in the PCA indicated long-term and short-term LC effects as principle components 1 and 2, accounting for $42 \%$ and $26 \%$ of transcriptional variation, respectively (Fig. 1B).

Consistent with the PCA of all expression values, we found a larger number of genes significantly ( $\mathrm{q}<0.01$, Bonferroni corrected) changed $24 \mathrm{~h}$ (long-term, 1021 genes in total, Fig. 2B, Table S3) after the shift from HC to LC conditions than $3 \mathrm{~h}$ (short-term, 552 genes in total, Fig. 2A, Table S3) after the shift. For 102 genes, the $24 \mathrm{~h}$ cultivation phase under $\mathrm{HC}$ conditions was not sufficient to fully recover the initial HC expression situation (Fig. 2C, Table S3) accounting for the difference between $\mathrm{HC} 0 \mathrm{~h}$ and $\mathrm{HC} 24 \mathrm{~h}$ in the PCA (Fig. 1B).

\subsection{Identification of $\mathrm{CO}_{2}$-dependent gene expression patterns}

To search for $\mathrm{CO}_{2}$-dependent gene expression patterns, we performed $K$-means clustering. As a result, 10 different clusters were generated (Fig. 3). Clusters 1, 2, 3, and 4 contained genes, which were characterized by short-term reduced transcript levels. Genes of clusters 5 and 6 showed a decline in transcript level during the $24 \mathrm{~h}$ LC treatment. Clusters 7 and 8 contained genes with rapid transcript accumulation after $3 \mathrm{~h} \mathrm{LC}$ conditions, while in cluster 9 transcript levels constantly increased until $24 \mathrm{~h}$ after LC shift. Cluster 10 contained genes that were specifically induced in expression $24 \mathrm{~h}$ after LC shift.

To functionally characterize these clusters, we tested them for enrichment of gene ontology (GO) terms and MapMan categories (Thimm et al., 2004) expecting similar enrichments with these independent methods. Among the clusters, which contained short-term (3 h after LC shift) down-regulated genes, we found cluster 2 enriched for genes of the GO terms photosynthesis, light reactions, and tetrapyrrole biosynthesis (Table S2) and cluster 3 enriched with terms related to vesicle-mediated transport. Clusters 5 and 6 contained genes with reduced abundance in long-term $\mathrm{CO}_{2}$ deprivation ( $24 \mathrm{~h}$ after LC shift). Cluster 5 showed an enrichment in the MapMan category DNA synthesis/chromatin structure (Table S1) and corresponding GO terms related to DNA replication (Table S2), while cluster 6 was enriched in genes belonging to GO terms and MapMan categories related to translation and protein biosynthesis (Table S2) and protein biosynthesis (Table S1), respectively. For cluster 7, which contained short-term LC-induced genes, we observed a significant enrichment of genes involved in the MapMan category photosynthesis: light reactions (Table S1). Among the clusters 8 , 9, and 10, which contained constantly and long-term LC-induced genes (Fig. 3), cluster 8 was significantly enriched for genes connected to photorespiration (Table S1). No significant enrichments were detected for cluster 9 and 10 . 


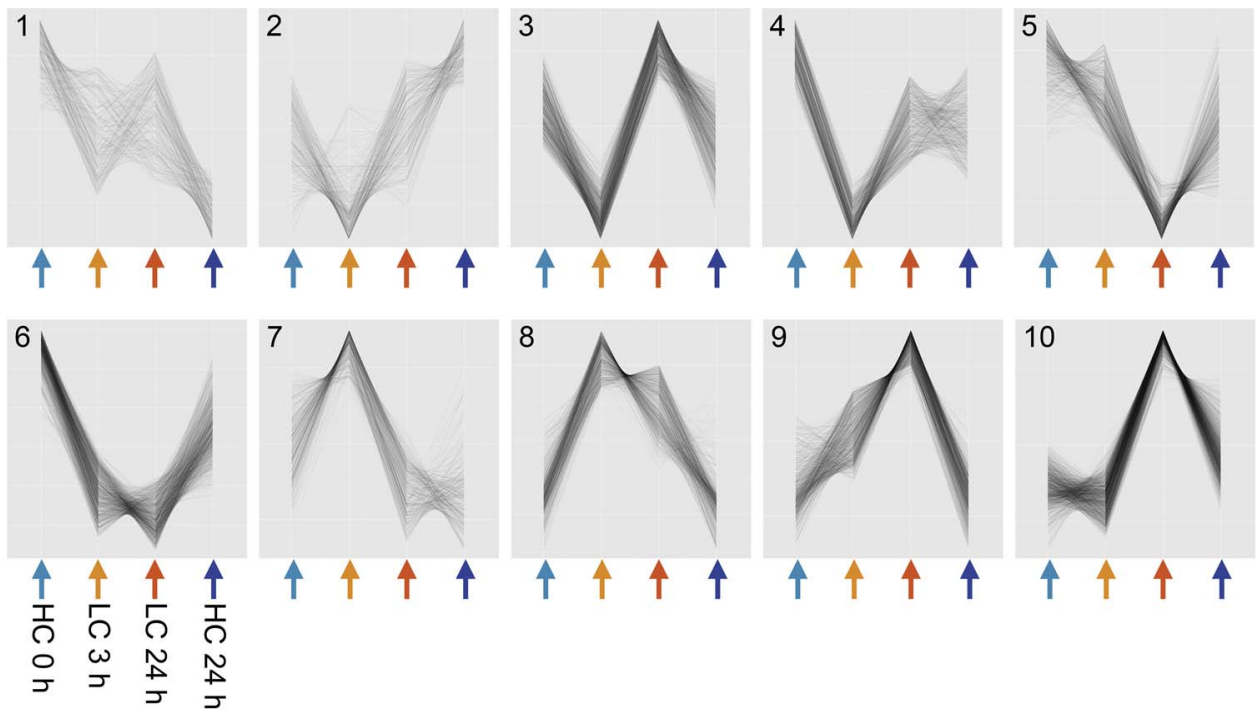

Fig. 3. Analysis of $\mathrm{CO}_{2}$-dependent expression patterns by $K$-means clustering. $K$-means cluster are shown in $z$-scores. Presented are 10 different clusters (1-10), representing different expression patterns upon changes in $\mathrm{CO}_{2}$ conditions. Color-coded arrows indicate different samples.
All genes encoding the photorespiratory enzymes (Rademacher et al., 2016) were strongly induced, ranging from 2.6-fold for hydroxypyruvate reductase (CMQ289C) to 53-fold for alanine:glyoxylate aminotransferase (CMS429C) $3 \mathrm{~h}$ after shift from HC to LC conditions. Though at a lower level compared to the LC $3 \mathrm{~h}$ value, these genes were still significantly up-regulated $24 \mathrm{~h}$ after the LC shift (Fig. 4, Table S5). Besides the photorespiratory genes, we also identified core elements of a hypothetical $\mathrm{C}_{4}$ cycle, funneling pyruvate into a PEP oxaloacetate cycle (Fig. 4, Table S5), significantly up-regulated during the LC treatment. Cluster 8 contained the genes encoding phosphoenolpyruvate carboxylase (PPC, CME095C) and phosphoenolpyruvate carboxykinase (PEPCK, CMN285C). With a 22-fold induction, pyruvate phosphate dikinase (PPDK, CMFO12C) was one of the 10 most upregulated genes $3 \mathrm{~h}$ after the $\mathrm{CO}_{2}$ shift. The transcript accumulation was even higher $24 \mathrm{~h}$ after LC shift. Accordingly, PPDK belonged to cluster 9. The pyruvate regenerating mechanism via malate dehydrogenase and
NADP-malic enzyme exists in C. merolae as both genes were expressed at $\mathrm{HC} 0 \mathrm{~h}$. Their expression however was significantly reduced in response to the LC shift (Fig. 4, Table S5).

\subsection{Bicarbonate transporters and carbonic anhydrases}

We furthermore employed the RNA-seq data set to search for possible components of a hypothetical CCM. We followed the rationale that candidate genes involved in an inducible CCM should be co-regulated with genes encoding proteins of the photorespiratory metabolism, as demonstrated for the green alga Chlamydomonas reinhardtii (C. reinhardtii) (Fang et al., 2012). Thus, we investigated clusters 8 and 9, which contained the majority of photorespiratory genes, for the occurrence of $\mathrm{HCO}_{3}{ }^{-}$transporters and CAs, which might participate in a hypothetical CCM. BlastP analyses identified two candidate proteins in C. merolae homologous to known $\mathrm{HCO}_{3}{ }^{-}$transport proteins in $C$. reinhardtii (Table S6). The protein CMN251C showed

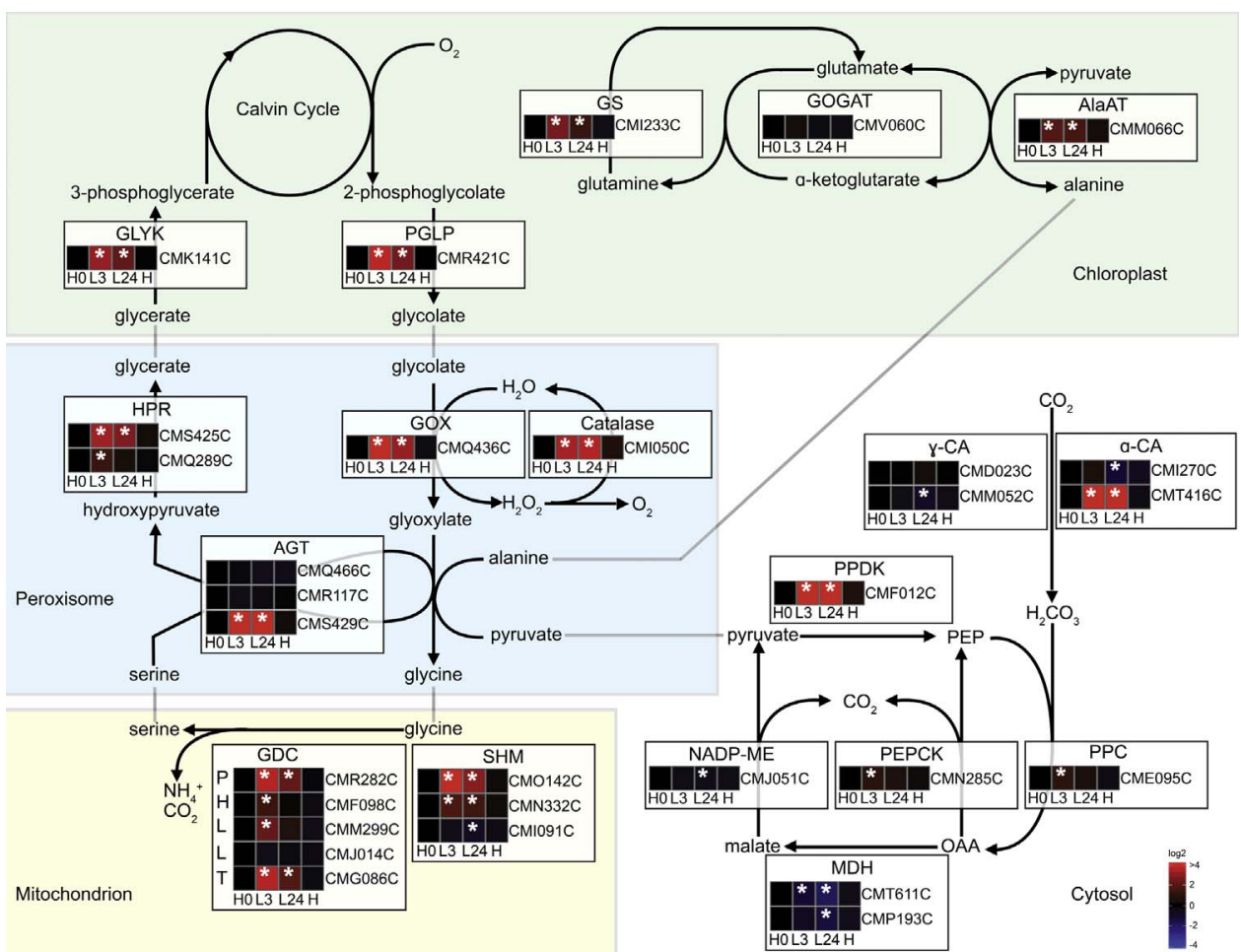

Fig. 4. The transcriptional response of genes involved in photorespiratory metabolism and $\mathrm{HCO}_{3}{ }^{-}$ homeostasis to changes in $\mathrm{CO}_{2}$ availability. The expression profiles of all genes involved in photorespiration and carbon sequestering from $\mathrm{HCO}_{3}{ }^{-}$are presented as $\log 2$-fold changes relative to their expression at sampling point $\mathrm{HO}$, and are illustrated as heat map. The sampling points are indicated by $\mathrm{H} 0$ (HC $0 \mathrm{~h}$ ), L3 (LC $3 \mathrm{~h}$ ), L24 (LC $24 \mathrm{~h}$ ), and H (HC $24 \mathrm{~h}$ ). All significant expression changes, at $\mathrm{q}<0.05$, are indicated by white asterisks. 


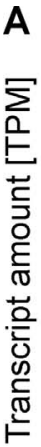

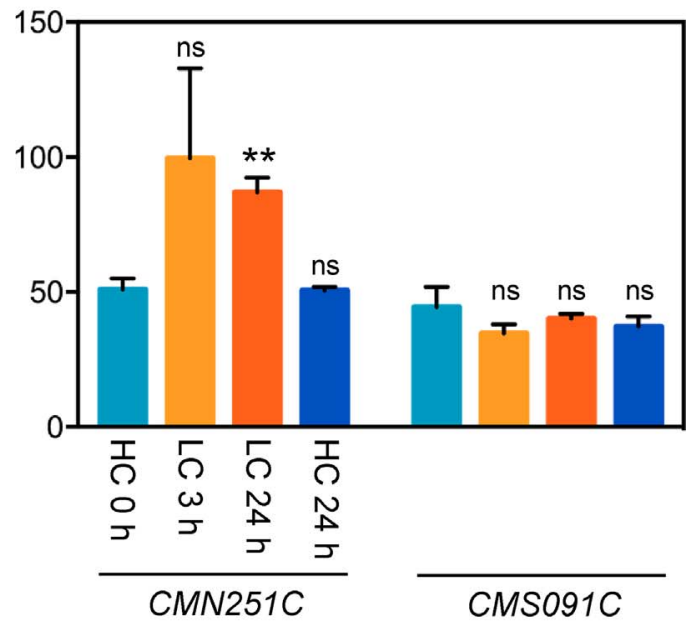

B

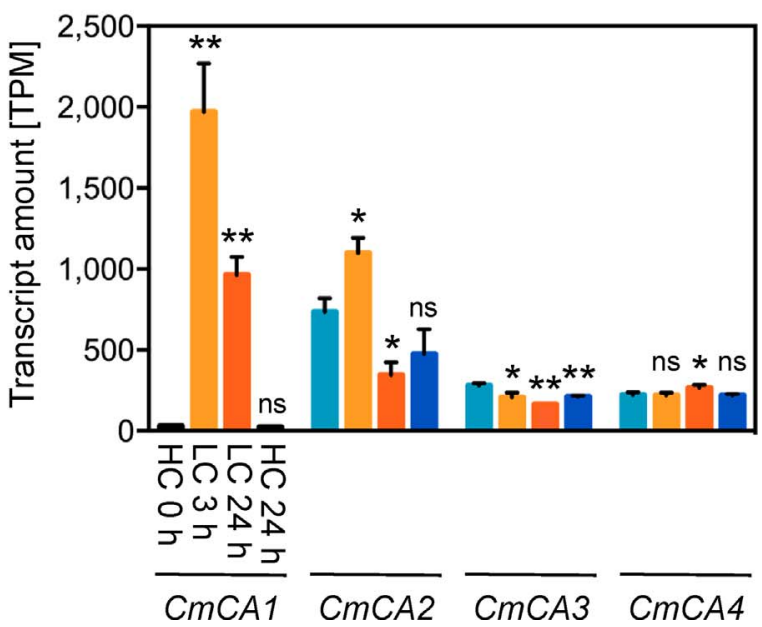

Fig. 5. Effect of changes in $\mathrm{CO}_{2}$ concentrations on transcript abundances of genes encoding hypothetical $\mathrm{HCO}_{3}{ }^{-}$transporters and carbonic anhydrases. A: $\mathrm{CO}_{2}$-dependent changes in transcript amounts of CMN251C and CMS091C, encoding hypothetical $\mathrm{HCO}_{3}{ }^{-}$ transporters. B. $\mathrm{CO}_{2}$-dependent changes in transcript amounts of CmCA1 (CMT416C), CmCA2 (CMI270C), CmCA3 (CMM052C), and CmCA4 (CMD023C). Transcript amounts are given in transcripts per million (TPM). Significant differences in relation to the $\mathrm{HC} 0 \mathrm{~h}$ value (paired $t$-test, two-tailed), are indicated as * $(\mathrm{P}<0.05)$ and $* *(\mathrm{P}<0.01)$, ns: not significant.

homology to the HIGH-LIGHT INDUCED GENE3 (HLA3) $\mathrm{HCO}_{3}{ }^{-}$transporter, which is induced under limiting $\mathrm{CO}_{2}$ conditions in C. reinhardtii (Tirumani et al., 2014). The protein CMS091C is homologous to the CHLOROPLAST ENVELOPE PROTEINS1 and 2 (CCP1 and CCP2), which showed $\mathrm{HCO}_{3}{ }^{-}$transporter function in C. reinhardtii (Tirumani et al., 2014). Expression of the gene CMN251C followed the same transcription pattern as the photorespiratory genes with a 2-fold transcript accumulation $3 \mathrm{~h}$ and $24 \mathrm{~h}$ after the shift to LC conditions (Fig. 5A, Table S5, cluster 8). In contrast, expression of CMS091C did not cluster together with the photorespiratory genes. The transcription pattern was not affected by the $\mathrm{CO}_{2}$ conditions (Fig. 5A, Table S5) and grouped in cluster 4.

BlastP analyses with known $\alpha$-, $\beta$-, and $\gamma$-CAs of the model plant Arabidopsis thaliana (A. thaliana) (Fabre et al., 2007) revealed four proteins homologous to CAs in C. merolae: CmCA1 (CMT416C), CmCA2 (CMI270C), CmCA3 (CMM052C), and CmCA4 (CMD023C). CmCA1 and CmCA2 were homologous to $\alpha$-CAs and CmCA3 and CmCA4 homologous to $\gamma$-CAs of $A$. thaliana (Table S7). Genes encoding $\alpha$-CAs showed $\mathrm{CO}_{2}$-dependent transcriptional dynamics (Fig. 5B, Table S5). Expression of $C m C A 1$ was most affected by the changing $\mathrm{CO}_{2}$ conditions. While under HC conditions the gene was only weakly expressed, we observed a 67-fold accumulation of CmCA1 transcript $3 \mathrm{~h}$ after the shift to LC conditions and a 33-fold increased value $24 \mathrm{~h}$ after the shift. The final $24 \mathrm{~h} \mathrm{HC}$ treatment lead to full recovery to the initial HC transcript value (cluster 8). Transcript amounts of CmCA2 increased 1.5-fold $3 \mathrm{~h}$ after the LC shift, went down to $50 \%$ of the initial HC value after $24 \mathrm{~h} \mathrm{LC}$ treatment and fully recovered to the HC default value after $24 \mathrm{~h} \mathrm{HC}$ conditions (cluster 7). In contrast, both $\gamma$-CAs, CmCA3 and CmCA4, were quite constantly expressed in $C$. merolae under HC and LC conditions (Fig. 5B, Table S5). The LC shift induced a slight reduction (0.7fold) in transcript abundances for $C m C A 3$ (cluster 6), while transcript amounts were slightly but significantly increased (1.2-fold) for CmCA4 $24 \mathrm{~h}$ after shift to LC conditions (cluster 10).

\subsection{Subcellular localization of CmCA1 and CmCA2}

On the basis of the LC-inducible expression of CmCA1 and CmCA2, we investigated the encoded proteins in more detail. An amino acid alignment showed an identity of $90 \%$ between both proteins. Interestingly, CmCA1 contained an additional $\mathrm{N}$-terminal extension of 109 amino acids length (Fig. S1). Closer investigation of this extension indicated the occurrence of a transmembrane region (23 amino acids length) predicted by the SOSUI tool (Hirokawa et al., 1998) and a mitochondrial target peptide predicted by TargetP (Emanuelsson et al., 2000) (Fig. S1). To determine in which subcellular compartment the $\alpha$ CAs of $C$. merolae reside, localization constructs were designed with an $\mathrm{N}$ - or C-terminal YFP-fusion to avoid masking of a hypothetical target peptide signal. In transiently transformed tobacco protoplasts YFP:CmCA1, YFP:CmCA2, and CmCA2:YFP were detected in the cytosol. For CmCA1:YFP the fluorescent signal was mostly detected around chloroplasts, indicating a possible attachment of CmCA1 to the chloroplast envelope (Fig. 6A). Transient expression of the YFP fusion proteins in $C$. merolae cells consistently revealed cytosolic localization of YFP:CmCA1, YFP:CmCA2, and CmCA2:YFP. In the case of CmCA1:YFP we observed YFP fluorescence at the interface between chloroplast and mitochondrion (Fig. 6B).

\section{Discussion}

The impact of reduced $\mathrm{CO}_{2}$ availability on the transcriptome of $C$. merolae was large. In the short-term ( $3 \mathrm{~h}$ after shift to LC conditions), $11 \%$ of all genes were changed in transcript abundance (Fig. 2), which is well in the range of a comparable study with $C$. reinhardtii $4 \mathrm{~h}$ after a shift from HC to LC conditions (Fang et al., 2012) and markedly different from the response of land plants (Eisenhut et al., 2017; Queval et al., 2012). Long-term ( $24 \mathrm{~h})$ LC treatment increased the number of significant changes (21\% of all genes changed, Fig. 2) in line with the larger variation captured in the PCA in the direction of long-term change compared to short-term change (Fig. 1B).

The three sampling time-points captured three pivotal phases in the responses of $C$. merolae to changes in $\mathrm{CO}_{2}$ availability: the initial counter response including up-regulation of photorespiration and a potential CCM, the long-term acclimation to reduced carbon in the metabolic system, and the resupply with carbon in the system. Intriguingly, the short-term high abundance response evident in the clusters 8 and 9 (Fig. 3) included not only the full set of genes encoding the enzymes of the photorespiratory cycle (derived from Rademacher et al., 2016), but also CAs and genes known to be involved in the $\mathrm{C}_{4}$ cycle (Table S5). This coordinated up-regulation (Fig. 4, Table S5) strongly underscores the importance of the photorespiratory pathway in C. merolae under $\mathrm{CO}_{2}$ concentrations present in its ecological niche, and points to a concerted transcriptional regulon of the photorespiratory genes. Among the potential transcriptional regulators changing in expression concomitantly in clusters 8 and 9 , the strongest changes in amplitude were present in putative histone de-acetylases (CMQ158C, CMG132C, Table S5), suggesting a potential role of epigenetic processes, in a gene similar to sigma factor B (CMR165C, Table 
A
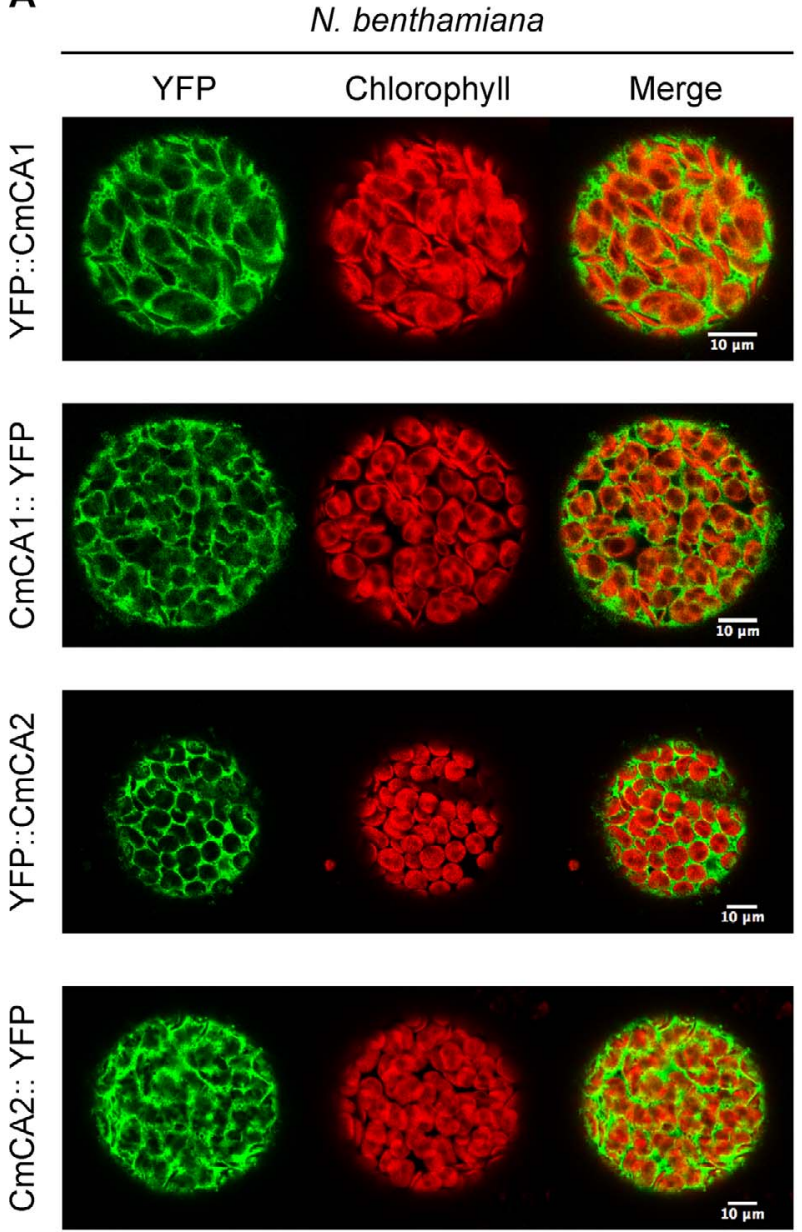

B
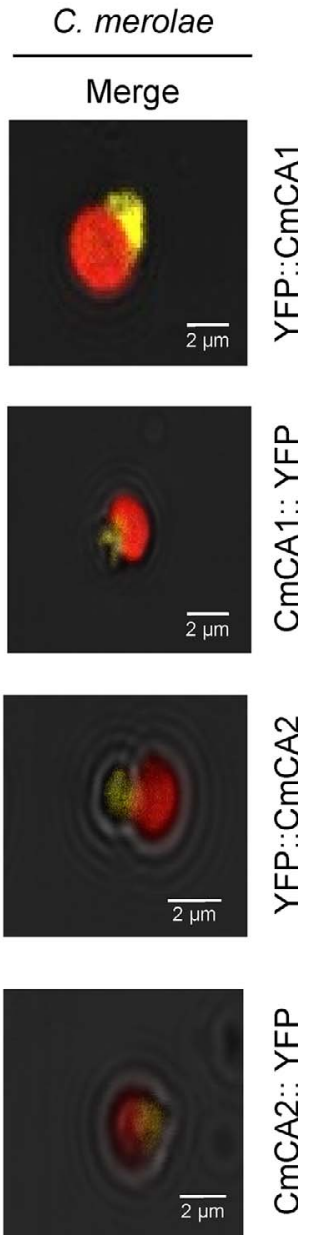

Fig. 6. Subcellular localization studies of carbonic anhydrases. CmCA1 (CMT416C) and CmCA2 (CMI270C) were Nand C-terminally fused with YFP, respectively. A. Fluorescent pictures of tobacco protoplast, transiently expressing YFP:CmCA1, CmCA1:YFP, YFP:CmCA2, and CmCA2:YFP fusion proteins, respectively. Pictures were recorded $48 \mathrm{~h}$ after infiltration of $N$. benthamiana leaves. B) Localization of CmCA1 and CmCA2 in C. merolae. Shown are merged pictures. The YFP signal is presented in green and yellow, respectively, chlorophyll autofluorescence in red. C. merolae cells were transformed $24 \mathrm{~h}$ before microscope analysis.
S5), which likely mediates chloroplast transcription, and in a regulator homologous to BRUTUS (CMM141C, Table S5), which controls the iron response transcription factor POPEYE in higher plants. Long-term carbon shortage at the LC $24 \mathrm{~h}$ time-point reduces growth (Rademacher et al., 2016) and consequently growth associated processes, such as DNA replication, the cell cycle, and protein biosynthesis were reduced in transcriptional abundance (Fig. 3: clusters 5 and 6, Table S1, Table S2). Reduction of transcriptional abundance for these processes has also been observed in carbon starved land plants (Brilhaus et al., 2016) and carbon starved C. reinhardtii cells (Brueggeman et al., 2012; Fang et al., 2012) pointing to a preserved ancient survival mechanism. During the response to LC, induced by low external $\mathrm{CO}_{2}$ in algae or by drought induced stomatal closure in land plants, the responses are however different. In both cases, the species initially respond with a reduction in the transcriptional investment into photosynthesis (Fig. 3, cluster 2) (Brilhaus et al., 2016). The long-term responses however present a different pattern. The land plant maintains the low expression levels of photosynthetic genes, while the red alga recovers its transcriptional investment. C. merolae naturally grows under LC conditions and presumably recovers photosynthetic transcript abundance because its acclimation response re-enables efficient photosynthesis. The resupply of abundant $\mathrm{CO}_{2}$ and presumably concomitant availability of fixed carbon mitigated the majority of changes induced by $24 \mathrm{~h}$ of LC status as is evident from the short distance between $\mathrm{HC} 0 \mathrm{~h}$ and $\mathrm{HC} 24 \mathrm{~h}$ points in the PCA (Fig. 1B), and from the overall pattern (Fig. 3). This mitigation of changes has also been observed in land plants upon resupply with water, which in turn caused resupply with carbon (Brilhaus et al., 2016). However, a low number of genes (Fig. 2C) did not return to the initial HC starting expression value after a $24 \mathrm{~h} \mathrm{HC}$ treatment. Among these, we detected the gene encoding catalase (CMIO50C), which participates in the photorespiratory metabolism. The ongoing significantly enhanced gene expression might indicate that this time period was not sufficient for $C$. merolae to fully return to the metabolic or energetic state of HC cells despite attaining a growth rate equal to before treatment (Rademacher et al., 2016).

Co-expression is a powerful tool to search for unknown components in functionally related processes. It was demonstrated for $C$. reinhardtii that genes involved in photorespiratory metabolism are co-regulated with genes involved in an inducible CCM (Fang et al., 2012) and a photorespiratory transporter was identified by co-expression analysis in higher plants (Bordych et al., 2013; Pick et al., 2013). Thus, we searched clusters 8 and 9, which contained photorespiratory genes, for candidates establishing a hypothetical CCM in C. merolae. We identified a hypothetical $\mathrm{HCO}_{3}{ }^{-}$transporter, two CAs, and enzymes constituting a PEPCK-type $\mathrm{C}_{4}$ cycle within the LC-inducible clusters 8 and 9 (Table S5). We suggest the following hypothetical model (Fig. 7) for the concerted action of these proteins to function as CCM in C. merolae:

In its natural habitat with an acidic $\mathrm{pH}$ the major form of $\mathrm{C}_{\mathrm{i}}$ is $\mathrm{CO}_{2}$, which diffuses into the red algal cell. The cytoplasmic CAs (CmCA2 and CmCA3, Fig. 6) convert the $\mathrm{CO}_{2}$ rapidly into $\mathrm{HCO}_{3}{ }^{-}$and thus intracellularly capture the $\mathrm{C}_{\mathrm{i}}$. Additionally, we identified the protein CMN251C, which shows homology to the LC-inducible $\mathrm{HLA} 3 \mathrm{HCO}_{3}{ }^{-}$ transporter from C. reinhardtii (Table S6). The protein is predicted to reside in the plasma membrane. Thus, CMN251C is a promising candidate for $\mathrm{C}_{\mathrm{i}}$ uptake under LC conditions in C. merolae. Photosynthesis in the red alga is most efficient at a low extracellular $\mathrm{pH}$ and decreases at a neutral $\mathrm{pH}$ (Zenvirth et al., 1985) calling into question whether $\mathrm{HCO}_{3}{ }^{-}$is the major $\mathrm{C}_{\mathrm{i}}$ species taken up from the external medium by $C$. 


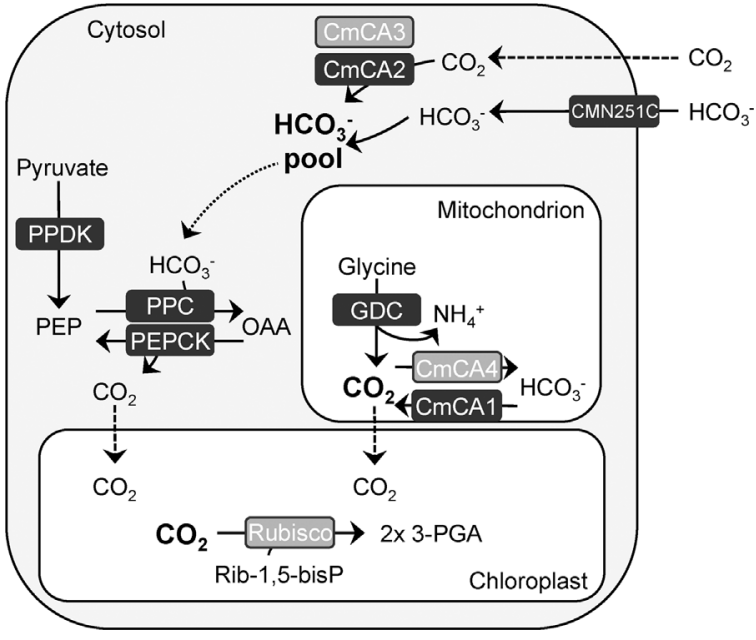

Fig. 7. Hypothetical model of a CCM in C. merolae. At low $\mathrm{pH}, \mathrm{C}_{\mathrm{i}}$ from the aquatic environment predominantly diffuses as $\mathrm{CO}_{2}$ into the cell, where it is converted by cytoplasmic CAs, CmCA2 and $\mathrm{CmCA} 3$, into $\mathrm{HCO}_{3}{ }^{-}$. Traces of environmental $\mathrm{HCO}_{3}{ }^{-}$are imported by the plasma membrane protein CMN251, which is homologous to HLA3. From the cytoplasmic pool, $\mathrm{HCO}_{3}{ }^{-}$is drained by the action of PPC, generating OAA and pulling more environmental $\mathrm{CO}_{2}$ into the cytoplasm. Decarboxylation of OAA by PEPCK allows stepwise release of $\mathrm{CO}_{2}$, which diffuses into the chloroplast for fixation by Rubisco. Besides this PEPCK-type $\mathrm{C}_{4}$ cycle, the concerted action of GDC and CmCA1 might function as a $\mathrm{CO}_{2}$ pump, by accumulating high amounts of $\mathrm{CO}_{2}$ in the mitochondrion, which is in close proximity to the chloroplast in the red algal cell. More details are presented in the text. Proteins, which are encoded by genes of the LC-inducible clusters 8 and 9 and presented in black boxes.

merolae. Possibly, CMN251C's role is the harvest of trace amounts of environmental $\mathrm{HCO}_{3}{ }^{-}$under $\mathrm{C}_{\mathrm{i}}$ limited condition or serving as a $\mathrm{CO}_{2}$ diffusion facilitator.

Genes encoding the components of a PEPCK-type $\mathrm{C}_{4}$ pathway (Fig. 4) were also contained in the photorespiratory gene cluster 8 . This might indicate a temporary $\mathrm{HCO}_{3}{ }^{-}$partitioning via PPC and PEPCK from the cytoplasmic $\mathrm{HCO}_{3}{ }^{-}$pool into organic acids, thereby increasing the concentration gradient and hence generating additional "pull" for $\mathrm{CO}_{2}$ entry into the cell. By the action of PEPCK the $\mathrm{CO}_{2}$ is released stepwise and allowed to diffuse into the chloroplast for fixation by Rubisco. A single cell $\mathrm{C}_{4}$ metabolism as proposed in diatoms is a distinct possibility. For example, in LC-acclimated Thalassiosira weissflogii cells, it was shown that repression of PPC activity by the inhibitor 3,3-dichloro-2-dihydroxyphosphinoylmethyl-2-propenoate resulted in a $90 \%$ decrease in the rate of photosynthesis, which could be overcome by HC (Reinfelder et al., 2004). For the model diatom Phaeodactylum tricornutum, the primary function of $\mathrm{C}_{4}$ metabolism might not be in $\mathrm{CO}_{2}$ fixation but rather play a role in dissipating energy and maintaining $\mathrm{pH}$ homeostasis (Haimovich-Dayan et al., 2013). Alternatively, the transient upregulation of PPC and PEPCK under $\mathrm{CO}_{2}$ limitation might have to do with overcoming short-term carbon limitation and gluconeogenesis from lipid stores and/or protein degradation.

The shift to LC conditions enhances the flux through the photorespiratory cycle in C. merolae (Rademacher et al., 2016). Accordingly, photorespiratory glycine cleavage in the mitochondrion by the GDC generates increased amounts of $\mathrm{CO}_{2}$ and $\mathrm{NH}_{3}$. The latter is likely protonated to $\mathrm{NH}_{4}{ }^{+}$at the $\mathrm{pH}$ of the mitochondrial matrix leading to an alkalization (Eriksson et al., 1996). A function in maintaining the $\mathrm{pH}$ homeostasis was suggested for the LC-inducible mitochondrial CAs in $C$. reinhardtii (Eriksson et al., 1996). In accordance, we postulate that CmCA1, which was almost exclusively expressed under LC conditions (Fig. 5B), fulfills this function in C. merolae. Interestingly, we observed in transiently transformed $C$. merolae cells the YFP fluorescence signal of a CmCA1:YFP protein at the interface between chloroplast and mitochondrion (Fig. 6B). A localization to the mitochondrial membrane is in accordance with the in silico prediction of a transmembrane helix and a mitochondrial targeting peptide in the $\mathrm{N}$-terminal extension specific for CmCA1 (Fig. S1). If CmCA1 was indeed attached to the mitochondrial membrane, its $\mathrm{CO}_{2}$ production might allow high $\mathrm{CO}_{2}$ accumulation in close proximity to the chloroplast. Thus, the mitochondrial $\mathrm{CO}_{2}$ provision by the concerted action of GDC and CmCA1 might serve as a $\mathrm{CO}_{2}$ pump for $\mathrm{CO}_{2}$ enrichment around Rubisco as part of a CCM. Alternatively, CmCA1's role may be the intracellular trapping of the high volume of $\mathrm{CO}_{2}$ released during photorespiratory glycine decarboxylation avoiding leakage of $\mathrm{CO}_{2}$ from the cell.

Clusters 8 and 9 also contained numerous genes encoding proteins of unknown function (Table S3). These LC-responsive proteins are potential candidates for involvement in the LC acclimation in C. merolae either to detoxify Rubisco oxygenation products or to enrich $\mathrm{CO}_{2}$ at the site of Rubisco.

Although the RNA-seq data only provide tantalizing suggestions about a CCM in the red alga and eventually cannot distinguish between a transport based CCM, a biochemical CCM, a mixed type as here suggested (Fig. 7), or an as of yet undiscovered mechanism, C. merolae has demonstrated higher apparent affinity to $\mathrm{CO}_{2}$ under $\mathrm{LC}$ conditions (Rademacher et al., 2016; Zenvirth et al., 1985), raising the possibility that one or more of the mechanism described above are functional.

\section{Conclusion}

In conclusion, the red alga $C$. merolae shows a distinct transcriptional response to reduction in $\mathrm{CO}_{2}$ availability. The $\mathrm{CO}_{2}$ limitation makes the cells reduce transcripts of protein biosynthesis and DNA replication when growth is stalled. As one strategy to acclimate to LC conditions, abundance of photorespiratory transcripts is uniformly upregulated. Additionally, transcripts constituting a hypothetical PEPCK $\mathrm{C}_{4}$ pathway, a $\mathrm{C}_{\mathrm{i}}$ transporter, and the mitochondrial CA CmCA1 are higher in abundance. Whether this represents a CCM footprint or a physiological adaptation in $\mathrm{pH}$ maintenance or energy homeostasis remains to be genetically and physiologically tested.

\section{Acknowledgements}

This work was supported by the Deutsche Forschungsgemeinschaft (WE 2231/8-2, WE2231/11-1). APMW acknowledges additional funding from EXC 1028. We thank the HHU BMFZ for technical support of the RNA-seq experiments.

\section{Appendix A. Supplementary data}

Supplementary data associated with this article can be found, in the online version, at http://dx.doi.org/10.1016/j.jplph.2017.06.014.

\section{References}

topGO: Enrichment Analysis for Gene Ontology. R package version 2.26.0.

Badger, M.R., Price, G.D., 2003. $\mathrm{CO}_{2}$ concentrating mechanisms in cyanobacteria: mo lecular components, their diversity and evolution. J. Exp. Bot. 54, 609-622.

Bauwe, H., Hagemann, M., Fernie, A.R., 2010. Photorespiration: players, partners and origin. Trends Plant Sci. J 15, 330-336.

Benjamini, Y., Hochberg, Y., 1995. Controlling the false discovery rate: a practical and powerful approach to multiple testing. J. R. Stat. Soc. Ser. B: Methodol. 57.

Benjamini, Y., Yekutieli, D., 2001. The control of the false discovery rate in multiple testing under dependency. Ann. Stat. 29, 1165-1188.

Bordych, C., Eisenhut, M., Pick, T.R., Kuelahoglu, C., Weber, A.P.M., 2013. Co-expression analysis as tool for the discovery of transport proteins in photorespiration. Plant Biol. 15, 686-693.

Breuers, F.K.H., Bräutigam, A., Geimer, S., Welzel, U., Stefano, G., Renna, L., Brandizzi, F., Weber, A.P.M., 2012. Dynamic remodeling of the plastid envelope membranes-a tool for chloroplast envelope in vivo localizations. Front. Plant Sci. 3, 1-10.

Brilhaus, D., Bräutigam, A., Mettler-Altmann, T., Winter, K., Weber, A.P., 2016 Reversible burst of transcriptional changes during induction of crassulacean acid metabolism in Talinum triangulare. Plant Physiol. 170, 102-122.

Brueggeman, A.J., Gangadharaiah, D.S., Cserhati, M.F., Casero, D., Weeks, D.P., Ladunga, I., 2012. Activation of the carbon concentrating mechanism by $\mathrm{CO}_{2}$ deprivation coincides with massive transcriptional restructuring in Chlamydomonas reinhardtii. Plant Cell 24, 1860-1875. 
Dereeper, A., Guignon, V., Blanc, G., Audic, S., Buffet, S., Chevenet, F., Dufayard, J.F., Guindon, S., Lefort, V., Lescot, M., Claverie, J.M., Gascuel, O., 2008. Phylogeny.fr: robust phylogenetic analysis for the non-specialist. Nucleic Acids Res. 36, W465-9.

Eisenhut, M., Kahlon, S., Hasse, D., Ewald, R., Lieman-Hurwitz, J., Ogawa, T., Ruth, W., Bauwe, H., Kaplan, A., Hagemann, M., 2006. The plant-like C2 glycolate cycle and the bacterial-like glycerate pathway cooperate in phosphoglycolate metabolism in cyanobacteria. Plant Physiol. 142, 333-342.

Eisenhut, M., Ruth, W., Haimovich, M., Bauwe, H., Kaplan, A., Hagemann, M., 2008. The photorespiratory glycolate metabolism is essential for cyanobacteria and might have been conveyed endosymbiontically to plants. Proc. Natl. Acad. Sci. U. S. A. 105, 17199-17204.

Eisenhut, M., Bräutigam, A., Timm, S., Florian, A., Tohge, T., Fernie, A.R., Bauwe, H., Weber, A.P., 2017. Photorespiration is crucial for dynamic response of photosynthetic metabolism and stomatal movement to altered $\mathrm{CO}_{2}$ availability. Mol. Plant. 10, 47-61.

Emanuelsson, O., Nielsen, H., Brunak, S., von Heijne, G., 2000. Predicting subcellular localization of proteins based on their N-terminal amino acid sequence. J. Mol. Biol. 300, 1005-1016.

Eriksson, M., Karlsson, J., Ramazanov, Z., Gardeström, P., Samuelsson, G., 1996 Discovery of an algal mitochondrial carbonic anhydrase: molecular cloning and characterization of a low- $\mathrm{CO}_{2}$-induced polypeptide in Chlamydomonas reinhardtii. Proc. Natl. Acad. Sci. U. S. A. 93, 12031-12034.

Fabre, N., Reiter, I.M., Becuwe-Linka, N., Genty, B., Rumeau, D., 2007. Characterization and expression analysis of genes encoding alpha and beta carbonic anhydrases in Arabidopsis. Plant Cell Environ. 30, 617-629.

Fang, W., Si, Y., Douglass, S., Casero, D., Merchant, S.S., Pellegrini, M., Ladunga, I., Liu, P., Spalding, M.H., 2012. Transcriptome-wide changes in Chlamydomonas reinhardtii gene expression regulated by carbon dioxide and the $\mathrm{CO}_{2}$-concentrating mechanism regulator CIA5/CCM1. Plant Cell 24, 1876-1893.

Giordano, M., Beardall, J., Raven, J.A., 2005. $\mathrm{CO}_{2}$ concentrating mechanism in algae: mechanisms, environmental modulation, and evolution. Annu. Rev. Plant Biol. 56, 99-131.

Grefen, C., Donald, N., Hashimoto, K., Kudla, J., Schumacher, K., Blatt, M.R., 2010. A ubiquitin-10 promoter-based vector set for fluorescent protein tagging facilitates temporal stability and native protein distribution in transient and stable expression studies. Plant J. 64, 355-365.

Hagemann, M., Kern, R., Maurino, V.G., Hanson, D.T., Weber, A.P.M., Sage, R.F., Bauwe, H., 2016. Evolution of photorespiration from cyanobacteria to land plants, considering protein phylogenies and acquisition of carbon concentrating mechanisms. J. Exp. Bot. 67, 2963-2976.

Haimovich-Dayan, M., Garfinkel, N., Ewe, D., Marcus, Y., Gruber, A., Wagner, H., Kroth, P.G., Kaplan, A., 2013. The role of C4 metabolism in the marine diatom Phaeodactylum tricornutum. New Phytol. 197, 177-185.

Hauser, T., Popilka, L., Hartl, F.U., Hayer-Hartl, M., 2015. Role of auxiliary proteins in Rubisco biogenesis and function. Nat. Plants 1, 15065.

Hirokawa, T., Boonćchieng, S., Mitaku, S., 1998. SOSUI: classification and secondary structure prediction system for membrane proteins. Bioinformatics 14, 378-379.

Imamura, S., Terashita, M., Ohnuma, M., Maruyama, S., Minoda, A., Weber, A.P., Inouye, T., Sekine, Y., Fujita, Y., Omata, T., Tanaka, K., 2010. Nitrate assimilatory genes and their transcriptional regulation in a unicellular red alga Cyanidioschyzon merolae: genetic evidence for nitrite reduction by a sulfite reductase-like enzyme. Plant Cell Physiol. 51, 707-717.

Kaplan, A., Reinhold, L., 1999. $\mathrm{CO}_{2}$ concentrating mechanisms in photosynthetic microorganisms. Annu. Rev. Plant. Physiol. Plant. Mol. Biol. 50, 539-570.

Krzywinski, M., Altman, N., 2014. Points of significance. Comparing samples-part I. Nat. Methods 11, 215-216.

Li, B., Dewey, C.N., 2011. RSEM: accurate transcript quantification from RNA-Seq data with or without a reference genome. BMC Bioinf. 12, 1-16.

Matsuzaki, M., Misumi, O., Shin-I, T., et al., 2004. Genome sequence of the ultrasmall unicellular red alga Cyanidioschyzon merolae 10D. Nature 428, 653-657.

McCarthy, D.J., Chen, Y., Smyth, G.K., 2012. Differential expression analysis of multifactor RNA-Seq experiments with respect to biological variation. Nucleic Acids Res. $40,4288-4297$.
Minoda, A., Sakagami, R., Yagisawa, F., Kuroiwa, T., Tanaka, K., 2004. Improvement of culture conditions and evidence for nuclear transformation by homologous recombination in a red alga, Cyanidioschyzon merolae 10D. Plant Cell Physiol. 45, $667-671$.

Nakamura, Y., Kanakagiri, S., Van, K., He, W., Spalding, M.H., 2005. Disruption of the glycolate dehydrogenase gene in the high- $\mathrm{CO}_{2}$-requiring mutant HCR89 of Chlamydomonas reinhardtii. Can. J. Bot. 83, 820-833.

Nozaki, H., Takano, H., Misumi, O., et al., 2007. A 100\%-complete sequence reveals unusually simple genomic features in the hot-spring red alga Cyanidioschyzon mer olae. BMC Biol. 5, 28.

Ohnuma, M., Yokoyama, T., Inouye, T., Sekine, Y., Tanaka, K., 2008. Polyethylene glycol (PEG)-mediated transient gene expression in a red alga, Cyanidioschyzon merolae 10D. Plant Cell Physiol. 49, 117-120.

Pérez-Rodríguez, P., Riaño-Pachón, D.M., Corrêa, L.G., Rensing, S.A., Kersten, B., Mueller-Roeber, B., 2010. PlnTFDB: updated content and new features of the plant transcription factor database. Nucleic Acids Res. 38, D822-7.

Pick, T.R., Bräutigam, A., Schulz, M.A., Obata, T., Fernie, A.R., Weber, A.P., 2013. PLGG1, a plastidic glycolate glycerate transporter, is required for photorespiration and defines a unique class of metabolite transporters. Proc. Natl. Acad. Sci. U. S. A. 110, 3185-3190.

Queval, G., Neukermans, J., Vanderauwera, S., Van Breusegem, F., Noctor, G., 2012. Day length is a key regulator of transcriptomic responses to both $\mathrm{CO}_{2}$ and $\mathrm{H}_{2} \mathrm{O}_{2}$ in Arabidopsis. Plant Cell Environ. 35, 374-387.

Rademacher, N., Kern, R., Fujiwara, T., Mettler-Altmann, T., Miyagishima, S.Y., Hagemann, M., Eisenhut, M., Weber, A.P., 2016. Photorespiratory glycolate oxidase is essential for the survival of the red alga Cyanidioschyzon merolae under ambient $\mathrm{CO}_{2}$ conditions. J. Exp. Bot. 67, 3165-3175.

Raven, J.A., Cockell, C.S., De La Rocha, C.L., 2008. The evolution of inorganic carbon concentrating mechanisms in photosynthesis. Philos. Trans. R. Soc. Lond. B. Biol. Sci. 363, 2641-2650.

Raven, J.A., Giordano, M., Beardall, J., Maberly, S.C., 2012. Algal evolution in relation to atmospheric $\mathrm{CO}_{2}$ : carboxylases, carbon-concentrating mechanisms and carbon oxidation cycles. Philos. Trans. R. Soc. Lond. B. Biol. Sci. 367, 493-507.

Reinfelder, J.R., Milligan, A.J., Morel, F.M., 2004. The role of the C4 pathway in carbon accumulation and fixation in a marine diatom. Plant Physiol. 135, 2106-2111.

Savir, Y., Noor, E., Milo, R., Tlusty, T., 2010. Cross-species analysis traces adaptation of Rubisco toward optimality in a low-dimensional landscape. Proc. Natl. Acad. Sci. U. S. A. $107,3475-3480$.

Seckbach, J., 1995. The first eukaryotic cells - acid hot-spring algae. J. Biol. Phys. 20, $335-345$.

Tcherkez, G.G., Farquhar, G.D., Andrews, T.J., 2006. Despite slow catalysis and confused substrate specificity: all ribulose bisphosphate carboxylases may be nearly perfectly optimized. Proc. Natl. Acad. Sci. U. S. A. 103, 7246-7251.

Thimm, O., Bläsing, O., Gibon, Y., Nagel, A., Meyer, S., Krüger, P., Selbig, J., Müller, L.A., Rhee, S.Y., Stitt, M., 2004. MAPMAN: a user-driven tool to display genomics data sets onto diagrams of metabolic pathways and other biological processes. Plant J. 37, 914-939.

Tirumani, S., Kokkanti, M., Chaudhari, V., Shukla, M., Rao, B.J., 2014. Regulation of CCM genes in Chlamydomonas reinhardtii during conditions of light-dark cycles in synchronous cultures. Plant Mol. Biol. 85, 277-286.

Uemura, K., Anwaruzzaman, Miyachi S., Yokota, A., 1997. Ribulose-1,5-bisphosphate carboxylase/oxygenase from thermophilic red algae with a strong specificity for $\mathrm{CO}_{2}$ fixation. Biochem. Biophys. Res. Comm. 233, 568-571.

Watanabe, S., Ohnuma, M., Sato, J., Yoshikawa, H., Tanaka, K., 2011. Utility of a GFP reporter system in the red alga Cyanidioschyzon merolae. J. Gen. Appl. Microbiol. 57, 69-72.

Yates, A., Akanni, W., Amode, M.R., et al., 2016. Ensembl 2016. Nucleic Acids Res. 44, D710-6.

Zelitch, I., Schultes, N.P., Peterson, R.B., Brown, P., Brutnell, T.P., 2009. High glycolate oxidase activity is required for survival of maize in normal air. Plant Physiol. 149, 195-204.

Zenvirth, D., Volokita, M., Kaplan, A., 1985. Photosynthesis and inorganic carbon accumulation in the acidophilic alga Cyanidioschyzon merolae. Plant Physiol. 77, 237-239. 